\title{
Sulfamethoxazole+Trimethoprim Induced Stevens- Johnson Syndrome in HIV Patient: A Case Report
}

\author{
Manjunath Gandage*, Gayathri Panumetcha, Neelkanthreddy Patil, Pooja Salimath, Harish Kumar, \\ Sheetal Ninne
}

Department of Pharmacy Practice, Basaweshwar Teaching and General Hospital, HKES's College of Pharmacy (MTRIPS), Sedam Road, Gulbarga, Karnataka, INDIA.

\begin{abstract}
Stevens-Johnson syndrome is mucocutaneous cellmediated hypersensitivity reaction which affects 2 to 3 cases per million. SJS is generally rare, but potentially lifethreatening and commonly drug induced. Trimethoprimsulfamethoxazole (TMP/SMX) is a widely prescribed antimicrobial for the management of several uncomplicated infections. It is commonly used for the treatment and prophylaxis of Pneumocystis Jirovecii Pneumonia (PCP) in the HIV-infected population. The adverse reaction to TMP/SMX is more frequent and severe in HIV-infected patients as compared to the general population. We report a case of 39-year-old male patient admitted into male medical free ward with a chief complaints of fever, difficulty in swallowing, skin lesions over the body from 4 days. Multiple well-defined erythematous macules, Erosion and crustations on angle of the mouth, Genital Involvement, Acute Conjuctivitis are observed. These clinical manifestations are observed after taking sulfamethoxazole + trimethoprim on $5^{\text {th }}$ day. Based on the above clinical investigations it is diagnosed as sulfamethoxazole + trimethoprim induced Stevens-Johnson syndrome.
\end{abstract}

Key words: Stevens-Johnson syndrome, Sulfamethoxazole + trimethoprim, HIV, ADR.

\section{INTRODUCTION}

Steven Johnson Syndrome (SJS) is characterized by sudden onset of erosion of mucous membranes (predominantly oral mucosa, lips and conjunctivae) with widespread blistering of the skin involving up to $10 \%$ of the body surface area. It is caused by several factors such as infections, drugs and malignancies. Trimethoprimsulfamethoxazole (TMP/SMX) is a widely prescribed antimicrobial for the management of several uncomplicated infections. In immunocompetent persons, the adverse reactions to TMP/SMX occur in approximately $1-3 \%$ of persons. In contrast, in HIV-infected population, a much higher incidence of adverse reactions has been reported with frequencies ranging from $40-$ $80 \%{ }^{1}$ Adverse reactions, although rare, still remain a major threat to the patient welfare. Stevens-Johnson Syndrome (SJS) is one such fatal drug reactions. "A new eruptive fever with stomatitis and opthalmia" was described as a severe variant of erythema multiforme and was termed by Steven and Johnson in 1922. By the 1940's it was commonly called as "Steven Johnson's Syndrome (SJS)". The concept of the spectrum of erythema multiforme has been widely accepted since that time. Although SJS is rare with an incidence of 0.05 to 2 persons per 1 million populations per year, it has significant impact on the public health in view of its high morbidity and mortality. ${ }^{2}$

\section{Case Discussion}

A 39-year-old male was admitted in the male medical free ward with the chief complaints of fever, difficulty in swallowing, skin lesions all over the body since 4 days. Multiple welldefined erythematous macules, Erosion and crustation on angle of the mouth, Genital Involvement and acute Conjuctivitis. On general examination his vitals were Pulse:80bpm, B.P:100/70mmHg, R.R:20/ min. Total Body Surface Area involvement of lesions was $10-30 \%$ BSA. On evaluation
DOI: 10.5530/ijopp.12.4.60

Address for correspondence: Prof. Manjunath Gandage, Assistant Professor, Department of Pharmacy Practice, HKES's College of Pharmacy (MTRIPS) Sedam Road, Gulbarga-585104, Karnataka, INDIA.

Phone no: +91 9632326585 Email Id: mggandage@gmail. com

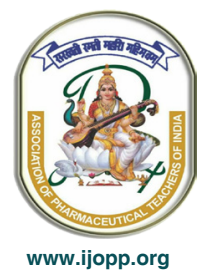


of medical history, the patient was diagnosed with $\mathrm{K} / \mathrm{C} / \mathrm{O}$ RVD two years. The patient is on ATT from 2 months. Patient started taking tab. Trimzole DS [sulf amethoxazole $(800 \mathrm{mg})+$ trimethoprim $(160 \mathrm{mg})]$ for PCP. Reaction occurred on $5^{\text {th }}$ day after taking tab. Trimzole, based on the above clinical investigations it is diagnosed as sulfamethoxazole +trimethoprim induced StevensJohnson syndrome. sulfamethoxazole+trimethoprim was discontinued. Patient was treated with Inj. Dexamethasone, Inj. Pheniramine maleate, Oraway paste, betamethasone+mupirocin cream. The reaction was assessed using casualty assessment scales. Finally, the patient was improved and was discharged for 9 days of hospital stay. The patient attenders were counselled regarding the condition of the patient. Various studies have shown that sulfamethoxazole/ trimethoprim (SMX/TMP) are most common drugs implicated in the causation of SJS. ${ }^{3}$ Early use of short-term dexamethasone therapy seems beneficial. Short term dexamethasone therapy on three consecutive days at an early stage of the reaction may benefit the patient. A recent systematic review has shown the beneficial effect of steroids. In our case, IV dexamethasone therapy proved to be effective. ${ }^{4} \mathrm{~A}$ causality analysis was done using the Naranjo's algorithm, ${ }^{5}$ WHO-UMC Scale and the present ADR is probable with a score of 5, WHO-UMC scale the ADR is probable/ likely, schumock and thornton assessed the ADR as probably preventable and the Hartwig and Siegel severity scale showed that the reaction was level 5 .

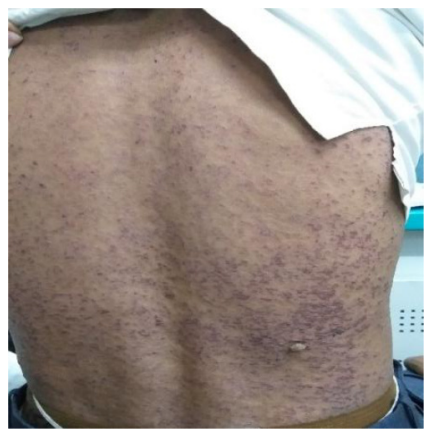

Maculopapular Rashes

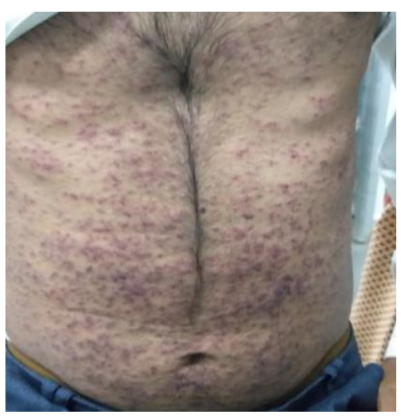

Erythematous macules

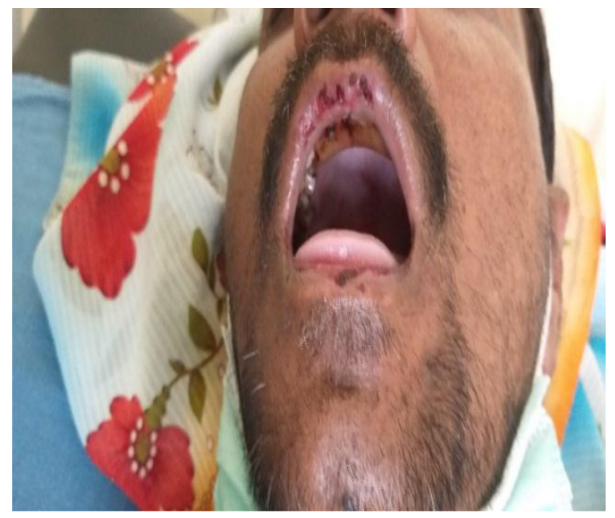

Crustations on the angle of the mouth

\section{Patient Alert Card}

Alert card was issued to the patient attenders and was advised to reveal this medication allergy to the other physicians before getting prescription from them to avoid this drug which causes SJS.

\section{Patient Consent Form}

Written informed consent form was taken from the patient for the presentation/publication of the case report.

\section{CONCLUSION}

Adverse drug reactions are an important concern. So, rigid ADR monitoring is necessary to ensure safety of drug therapy. Care should be taken while prescribing the drug Sulfamethoxazole+Trimethoprim in HIV patients with opportunistic infections as it might be a risk factor for causing SJS. Patient and attenders should be counselled about the possible side effects. Therefore, TMP/SMX must NOT be rechallenged so as to avoid further fatal reactions. Early diagnosis helps to prevent secondary infections and subsequent complications. Pharmacovigilance which deals with the identification, assessment and prevention of ADRs can help in providing continuous information on medication safety and appropriate use.

\section{ACKNOWLEDGEMENT}

We would like to thank HOD, Department of Medicine for their support.

\section{CONFLICT OF INTEREST}

None.

\section{ABBREVIATIONS}

TMP/ SMX: Sulfamethoxazole+trimethoprim; 
SJS: Stevens-Johnson syndrome; HIV: Human

Immunodeficiency Virus; ADR: Adverse Drug Reaction.

\section{SUMMARY}

- SJS is generally rare, but potentially life threatening and commonly drug induced. Patient should be monitored while prescribing Sulfamethoxazole+Trimethoprim drug in HIV patients with opportunistic infections as it might be a risk factor for causing SJS.

- Red Alert card should be issued to the patient.

\section{REFERENCES}

1. Taqi SA, Zaki SA, Rajasab NA, Lateef BS. Trimethoprim-sulfamethoxazoleinduced Steven Johnson syndrome in an HIV-infected patient. Indian Journal of Pharmacology. 2012;44(4):533-5.

2. Manjunath G, Sheetal N, Pooja S, Neelkantreddy P, Snehasri Y. Carbamazepine Induced Stevens - Johnson Syndrome: A Case Report. IJOPP. 2018;11(3):164-6.

3. Harr T, French LE. Toxic epidermal necrolysis and stevens-Johnson syndrome. Orphanet Journal of Rare Disease. 2010;5(1):39.

4. Sew KT, Yong KT. Profile and Pattern of Stevens - Johnson syndrome and Toxic Epidermal Necrolysis in a General Hospital in Singapore: Treatment Outcomes. Acta Derm Venereol. 2011;91(1):62-6.

5. Srinivasan R, Ramya G. Adverse Drug Reaction - Causality Assessment. IJRPC. 2011;1(3):606-12. 